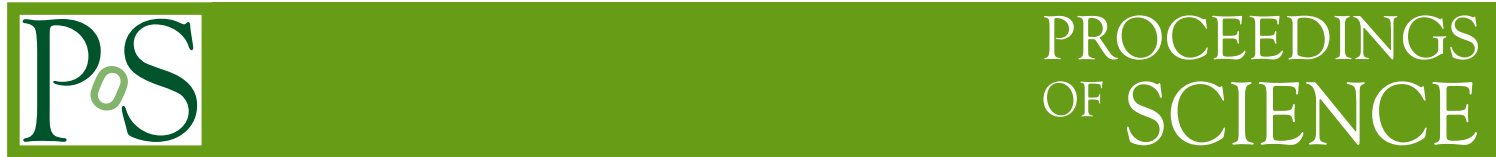

\title{
Probing the Nature of Cosmic Acceleration
}

\section{Yun Wang}

Homer L. Dodge Department of Physics \& Astronomy, Univ. of Oklahoma, 440 W Brooks St., Norman, OK 73019

E-mail: wang@nhn.ou.edu

The cause for the observed acceleration in the expansion of the universe is unknown, and referred to as "dark energy" for convenience. Dark energy could be an unknown energy component, or a modification of Einstein's general relativity. This dictates the measurements that are optimal in unveiling the nature of dark energy: the cosmic expansion history, and the growth history of cosmic large scale structure. Type Ia supernovae, galaxy clustering, and weak lensing are generally considered the most powerful observational probes of dark energy. Due to page limit, I will only examine Type Ia supernovae and galaxy clustering as dark energy probes, and discuss the recent results and future prospects.

VIII International Workshop on the Dark Side of the Universe,

June 10-15, 2012

Rio de Janeiro, Brazil

\footnotetext{
*Speaker.
} 

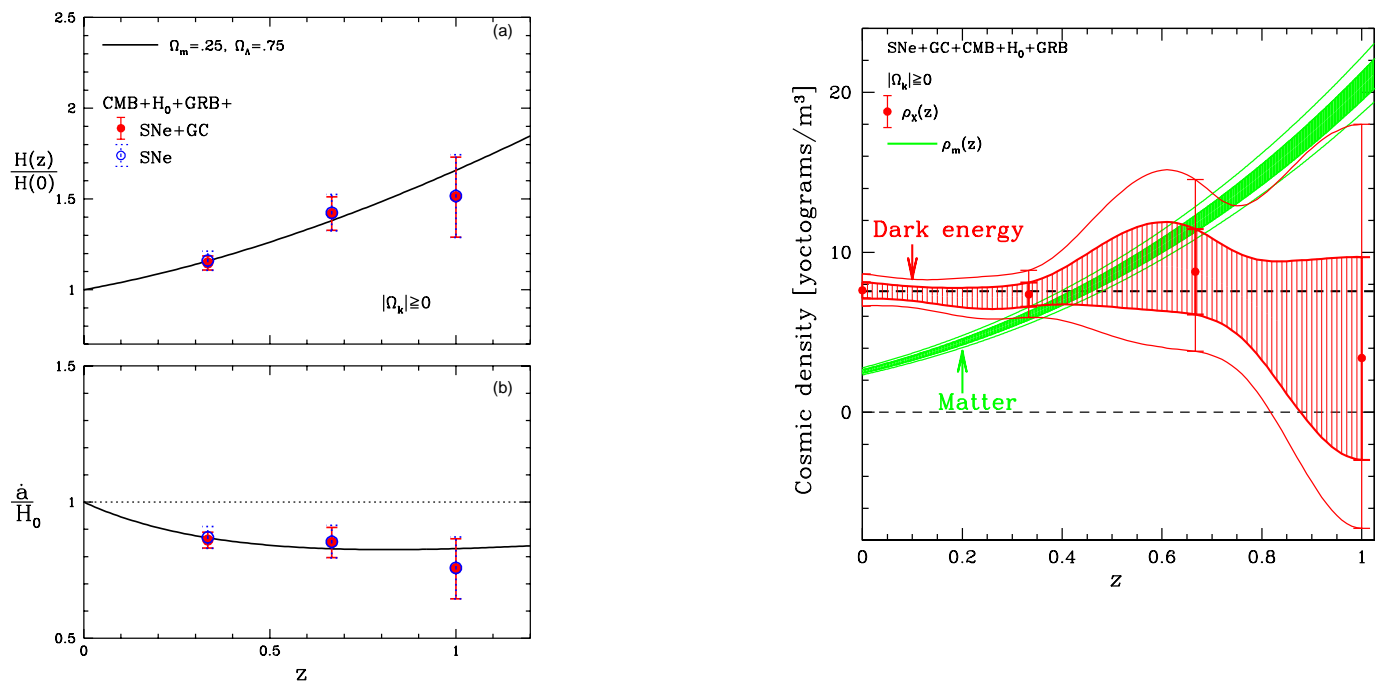

Figure 1: Left panel: Expansion history of the universe measured from current data [7]. Data used: Cosmic microwave background anisotropy (CMB) data from WMAP 7 year observations [8]; 472 Type Ia supernovae (SNe Ia) (compiled by [9], including data from the Supernova Legacy Survey (SNLS) [9], the Hubble Space Telescope (HST) [10], as well as nearby SNe Ia [36]); galaxy clustering measurements from Sloan Digital Sky Survey (SDSS) Luminous Red Galaxies (LRGs) [11], 69 Gamma Ray Bursts [14], and the latest Hubble constant $\left(H_{0}\right)$ measurement using the Hubble Space Telescope (HST) [16]. Right panel: Dark energy density as a function of redshift measured from the same data.

\section{Introduction}

Solving the mystery of the observed cosmic acceleration [1] is one of the most important challenges in cosmology today. Current observational data are not sufficient for differentiating two likely explanations for the observed cosmic acceleration: an unknown energy component (dark energy, e.g., [2]), and the modification of general relativity (modified gravity, e.g., [3, 4]). Refs.[5, $6]$ contains reviews with more complete lists of references of theoretical models.

The evidence for cosmic acceleration has strengthened over time. The expansion history of the universe is described by the Hubble parameter, $H(t)=(d \ln a / d t)=\dot{a} / a$, where $a(t)$ is the cosmic scale factor, and $t$ is cosmic time. The cosmological redshift, $z \equiv 1 / a(t)-1$, is usually used as the indicator for cosmic time, because it can be measured for a given astrophysical object. Fig.1 (left panel) shows the Hubble parameter $H(z)$, as well as $\dot{a}$, measured from current observational data [7]. Fig.1 (right panel) shows the corresponding dark energy density function $\rho_{X}(z)$ [7]. A cosmological constant (and no modification of gravity) continues to be consistent with data, but the uncertainties are large (see Fig.1, right panel). Given our ignorance of the true nature of dark energy, and the theoretical difficulties of explaining a tiny but non-vanishing cosmological constant using known physics, we need to be open minded in exploring and constraining alternative explanations.

Type Ia supernovae (SNe Ia), galaxy clustering (GC), and weak lensing (WL) are generally considered the most powerful observational probes of dark energy. SNe Ia provide a measurement of $H(z)$ derived from luminosity distances of SNe Ia. GC provides direct measurements of $H(z)$, $D_{A}(z)$, as well as the growth rate of cosmic large scale structure, $f_{g}(z)$. WL provides measurements of $H(z)$ and the growth factor $G(z)$ (related to $f_{g}(z)$ via a derivative) [12]. It is important to utilize 
all three methods, as they have different systematic errors. Furthermore, GC and WL constrain different aspects in the modification of gravity; both are required to achieve a robust test of gravity.

Clusters of galaxies provide an independent and complementary method to probe dark energy [13]. Other methods, e.g., using gamma-ray bursts, old red galaxies, or radio galaxies [15] provide additional cross-checks on dark energy constraints. CMB data and independent measurements of $H_{0}$ are required to break the degeneracy between dark energy and cosmological parameters (see e.g. $[17,7])$, hence are important as well in constraining dark energy.

Due to the page limit, I will focus on SNe Ia and GC, as these have yielded the strongest direct constraints on dark energy to date. I will first discuss the simplest general guidelines for probing dark energy, then SNe Ia and GC as dark energy probes respectively, and conclude with a brief summary of current status and future prospects of dark energy observational projects.

\section{Probing dark energy and testing gravity}

Because of the existence of two possible explanations, dark energy and modified gravity, for the observed cosmic acceleration, it is critical for us to recognize that we need to measure two functions of cosmic time from observational data: the expansion history of the universe, $H(z)$, and the growth rate of cosmic large scale structure, $f_{g}(z)$. Modified gravity models can give identical $H(z)$ as a dark energy model by design, but the growth rate $f_{g}(z)$ is likely different in these models compared to dark energy models. The precise and accurate measurement of $H(z)$ and $f_{g}(z)$ from observational data will allow us to probe the true nature of cosmic acceleration [19].

Dark energy is often parameterized by a linear equation of state $w_{X}(a)=w_{0}+w_{a}(1-a)$ [20]. Because of our ignorance of the nature of dark energy, it is important to make model-independent constraints by measuring the dark energy density $\rho_{X}(z)$ [or the expansion history $H(z)$ ] as a free function of cosmic time. Measuring $\rho_{X}(z)$ has advantages over measuring dark energy equation of state $w_{X}(z)$ as a free function; $\rho_{X}(z)$ is more closely related to observables, hence is more tightly constrained for the same number of redshift bins used [21,22]. Note that $\rho_{X}(z)$ is related to $w_{X}(z)$ as follows [21]:

$$
\frac{\rho_{X}(z)}{\rho_{X}(0)}=\exp \left\{\int_{0}^{z} \mathrm{~d} z^{\prime} \frac{3\left[1+w_{X}\left(z^{\prime}\right)\right]}{1+z^{\prime}}\right\}
$$

Hence parametrizing dark energy with $w_{X}(z)$ implicitly assumes that $\rho_{X}(z)$ does not change sign in cosmic time. This precludes whole classes of dark energy models in which $\rho_{X}(z)$ becomes negative in the future ("Big Crunch" models, see [23] for an example)[24]. If the present cosmic acceleration is caused by dark energy,

$$
E(z) \equiv \frac{H(z)}{H_{0}}=\left[\Omega_{m}(1+z)^{3}+\Omega_{k}(1+z)^{2}+\Omega_{X} X(z)\right]^{1 / 2}
$$

where $X(z) \equiv \rho_{X}(z) / \rho_{X}(0) . H_{0}=H(z=0)$ is the Hubble constant. $\Omega_{m}$ and $\Omega_{X}$ are the ratios of the matter and dark energy density to the critical density $\rho_{c}^{0}=3 H_{0}^{2} /(8 \pi G)$, and $\Omega_{k}=-k / H_{0}^{2}$ with $k$ denoting the curvature constant. Consistency of Eq.(2.2) at $z=0$ requires that $\Omega_{m}+\Omega_{k}+\Omega_{X}=1$. Once $E(z)$ is specified, the evolution of matter density perturbations on large scales, $\delta^{(1)}(\mathbf{x}, t)=$ 
$D_{1}(t) \delta(\mathbf{x})$ is determined by solving the following equation for $D_{1}=\delta^{(1)}(\mathbf{x}, t) / \delta(\mathbf{x}){ }^{1}$

$$
D_{1}^{\prime \prime}(\tau)+2 E(z) D_{1}^{\prime}(\tau)-\frac{3}{2} \Omega_{m}(1+z)^{3} D_{1}=0,
$$

where primes denote $d / d\left(H_{0} t\right)$. The linear growth rate

$$
f_{g}(z) \equiv d \ln D_{1} / d \ln a .
$$

In the simplest alternatives to dark energy, the present cosmic acceleration is caused by a modification to general relativity. Ref.[26] contains examples of studies of observational signatures of modified gravity models. A worked example is the DGP gravity model [4, 27], which can be described by a modified Friedmann equation ${ }^{2}$. The right panel of Fig.12 shows a DGP model that gives identical $H(z)$ as a dark energy model, but gives significantly different $f_{g}(z)$ [19].

\section{Type Ia supernovae as dark energy probe}

The use of Type Ia supernovae ( $\mathrm{SNe}$ Ia) is the best established method for probing dark energy, since this is the method through which cosmic acceleration has been discovered [1]. This method is independent of the clustering of matter ${ }^{3}$, and can provide a robust measurement of $H(z)$ [30] through the measured luminosity distance as a function of redshift, $d_{L}(z)=(1+z) r(z)$, where the comoving distance $r(z)$ from the observer to redshift $z$ is given by

$$
\begin{aligned}
& r(z)=c H_{0}^{-1}\left|\Omega_{k}\right|^{-1 / 2} \operatorname{sinn}\left[\left|\Omega_{k}\right|^{1 / 2} \Gamma(z)\right], \\
& \Gamma(z)=\int_{0}^{z} \frac{d z^{\prime}}{E\left(z^{\prime}\right)}, \quad E(z)=H(z) / H_{0}
\end{aligned}
$$

where $\operatorname{sinn}(x)=\sin (x), x, \sinh (x)$ for $\Omega_{k}<0, \Omega_{k}=0$, and $\Omega_{k}>0$ respectively.

Type Ia as standard candles. A SN Ia is a thermonuclear explosion that completely destroys a carbon/oxygen white dwarf near the Chandrasekher limit of $1.4 M_{\odot}$. This is the reason SNe Ia are so uniform in peak luminosity. The first challenge to overcome when using $\mathrm{SNe}$ Ia as cosmological standard candles is properly incorporating the intrinsic scatter in SN Ia peak luminosity. The usual calibration of SNe Ia reduces the intrinsic scatter in SN Ia peak luminosity (Hubble diagram dispersion) to about 0.16 mag $[31,32]$. The calibration techniques used so far are based on one observable parameter, the lightcurve width, which can be parametrized either as $\Delta m_{15}$ (decline in magnitudes for a SN Ia in the first 15 days after $B$-band maximum, see [31]), or a stretch factor (which linearly scales the time axis, see [33]). The lightcurve width is associated with the amount

\footnotetext{
${ }^{1}$ Note that we have assumed that dark energy and dark matter are separate, which is true for the vast majority of dark energy models that have been studied in the literature. If dark energy and dark matter are coupled (a more complicated possibility), or if dark energy and dark matter are unified (unified dark matter models), Eq.(2.3) would need to be modified accordingly. Ref.[25] found the first strong evidence for the separation of dark energy and dark matter by ruling out a broad class of so-called unified dark matter models. They showed that these models produce oscillations or exponential blowup of the dark matter power spectrum inconsistent with observation.

${ }^{2}$ The validity of the DGP model has been studied by [28].

${ }^{3}$ Galaxy peculiar velocities from large-scale supernova surveys can be used to probe dark energy [29].
} 

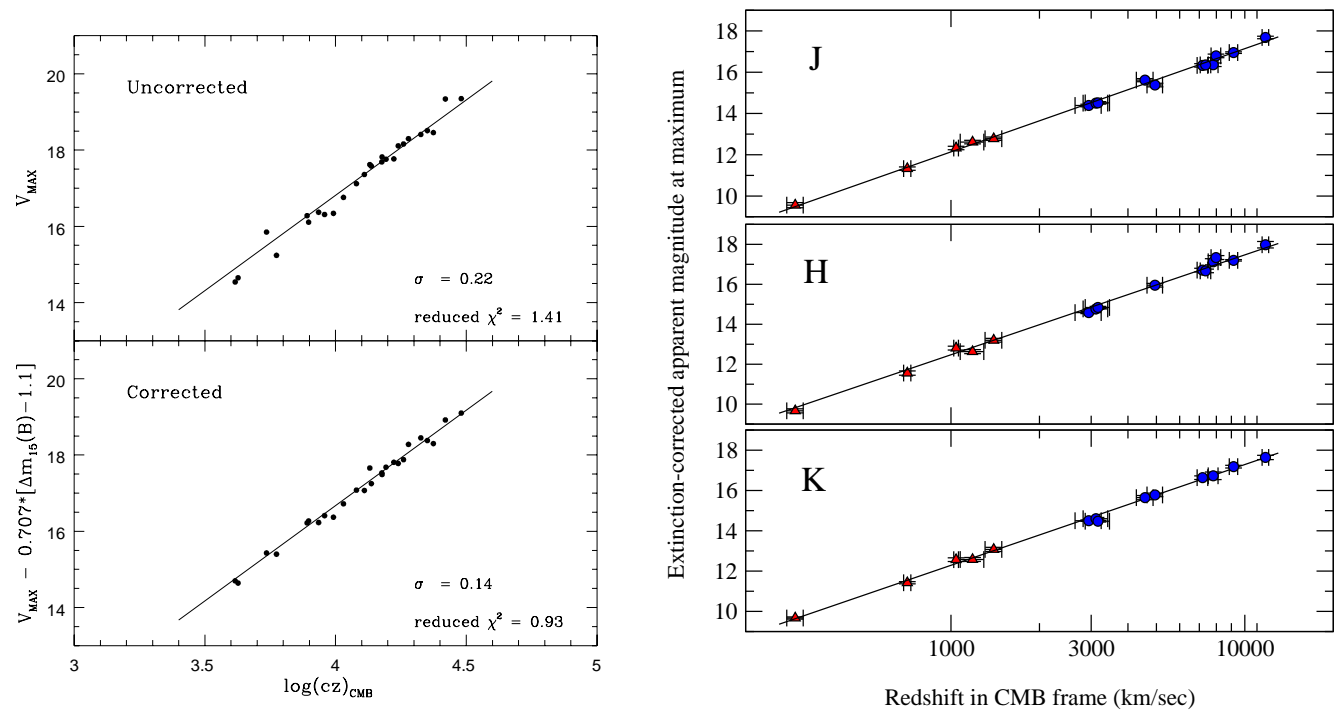

Figure 2: Left panel: Hubble diagrams showing $26 \mathrm{SNe}$ Ia with $B_{\max }-V_{\max } \leq 0.20$ from the Calan/Tololo sample [36]. This sample provided half of the data for the discovery of the cosmic acceleration in 1998 [1]. The solid lines indicate Hubble's law; perfect standard candles (with $\sigma=0$ ) fall on these lines. Right panel: Hubble diagrams of $\mathrm{SNe}$ Ia in the NIR bands. Note that these SNe Ia have only been corrected for dust extinction; no corrections have been made for lightcurve width. [46]

of ${ }^{56} \mathrm{Ni}$ produced in the $\mathrm{SN}$ Ia explosion, which in turn depends on when the carbon burning makes the transition from turbulent deflagration to a supersonic detonation [34]. There may be additional physical parameters associated with SN Ia lightcurves or spectra [35] that can further improve the calibration of SNe Ia. Fig.2 (left panel) shows a historic example of the homogeneity of SNe Ia [36].

Systematic effects of SNe Ia as dark energy probe. The main systematic effects of SNe Ia as a dark energy probe are: extinction by normal [37] or gray dust [38] $]^{4}$, weak lensing amplification by cosmic large scale structure [39], and possible evolution in the peak luminosity of SNe Ia.

Recent data show that the apparent dust extinction of SNe Ia is very different from the typical extinction law due to Milky Way dust, possibly due to the mixing of intrinsic SN Ia color variation with dust extinction, or variations in the properties of dust [42]. The extinction by dust can be corrected using multi-band imaging data, especially near infrared (NIR) observations of SNe, since dust extinction decreases with wavelength.

The weak lensing amplification of SNe Ia by cosmic large scale structure can be modeled by a universal probability distribution function for weak-lensing amplification based on the measured matter power spectrum [43]. The effect of weak lensing on the SN Ia data can be minimized through flux-averaging [44]. Figs. 3 shows the $2 \mathrm{D}$ marginalized contours of $\left(w, \Omega_{m}, \mathscr{M}\right)$ (where $\left.\mathscr{M}\right)$ is a nuisance parameter), assuming a constant equation of state for dark energy, $w$, and a flat universe.

\footnotetext{
${ }^{4}$ Gray dust, consisting of large dust grains, is difficult to detect by its reddening and could mimic the effect of dark energy [38]. Gray dust can be constrained quantitatively by the Cosmic Far Infrared Background [40], with no evidence found in favor of gray dust so far. Supernova flux correlation measurements can be used in combination with other lensing data to infer the level of dust extinction, and provide a viable method to eliminate possible gray dust contamination in SN Ia data [41].
} 

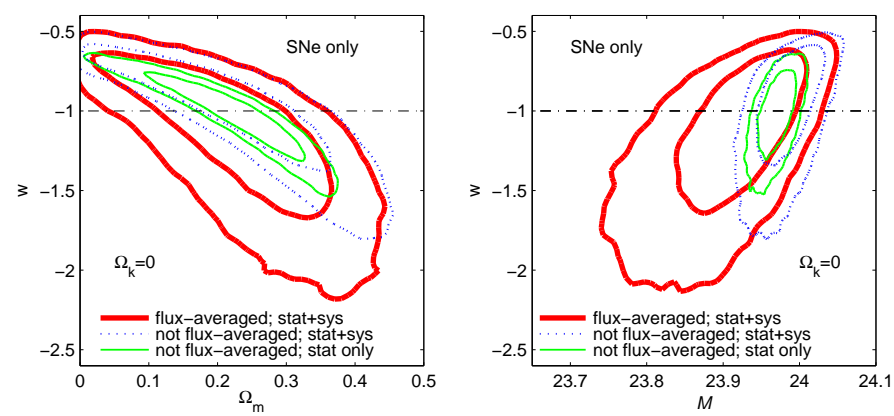

Figure 3: The 2D marginalized contours of $\left(w, \Omega_{m}, \mathscr{M}\right)$ for $\mathrm{SNe}$ data compiled by [9] (with and without fluxaveraging), assuming a flat universe. The contours are at $68 \%$ and $95 \%$ confidence levels. [7]
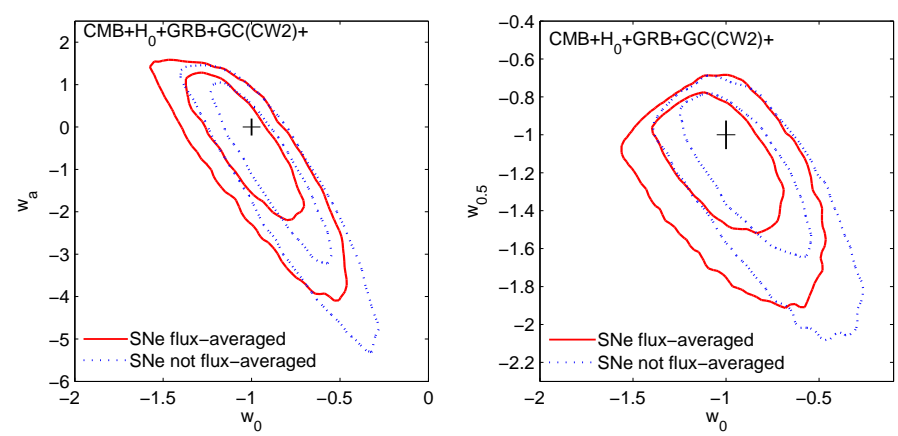

Figure 4: The 2D marginalized contours of $\left(w_{0}, w_{a}\right)$ and $\left(w_{0}, w_{0.5}\right)$ for $\mathrm{SNe}$ data (with and without flux-averaging) combined with galaxy clustering (CW2), CMB, $H_{0}$, and GRB data (same data as in Fig.1). The contours are at $68 \%$ and 95\% confidence levels. [7]

Note that the inclusion of systematic errors of SNe leads to significantly larger uncertainties in estimated parameters, compared to when only statistical errors of SNe are included [7]. Clearly, flux-averaging (thick solid lines) leads to larger errors on dark energy and cosmological parameters if only SN Ia data are used. However, when other data are added, flux-averaging leads to smaller errors on dark energy (see Figs.4-5) because flux-averaging increases the concordance of SNe Ia with other data.

The evolution in SN Ia peak luminosity could arise due to progenitor population drift, since the most distant $\mathrm{SNe}$ Ia come from a stellar environment very different (a much younger universe) than that of the nearby SNe Ia. However, with sufficient statistics, we can subtype SNe Ia and compare SNe Ia at high redshift and low redshift that are similar in both lightcurves and spectra, thus overcoming the possible systematic effect due to progenitor population drift [45].

Optimized observations of SNe Ia. NIR observations of SNe Ia provide additional strong advantages beyond being relatively dust-free. SNe Ia are better standard candles at NIR wavelengths compared to the optical wavelengths [46, 47, 48]. The right panel of Fig.2 shows the Hubble diagram of SNe Ia in the NIR, without the usual lightcurve width correction. The smaller intrinsic dispersion of SN Ia peak luminosity in the NIR can be explained by the theoretical modeling of SN Ia lightcurves using time-dependent multi-group radiative transfer calculations (Kasen 2006 [49]). Fig.6 shows the dispersion in peak magnitude (measured at the first lightcurve maximum) as 

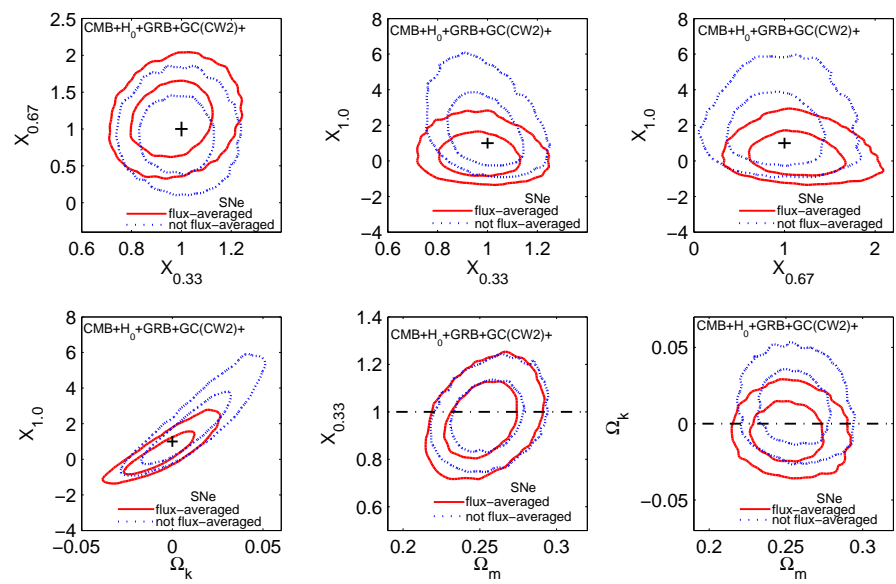

Figure 5: The 2D marginalized contours of $\left(X_{0.33}, X_{0.67}, X_{1.0}, \Omega_{m}, \Omega_{k}\right)$ for SNe data (with and without flux-averaging) combined with galaxy clustering (CW2), CMB, $H_{0}$, and GRB data (same data as in Fig.1). The contours are at $68 \%$ and 95\% confidence levels. [7]

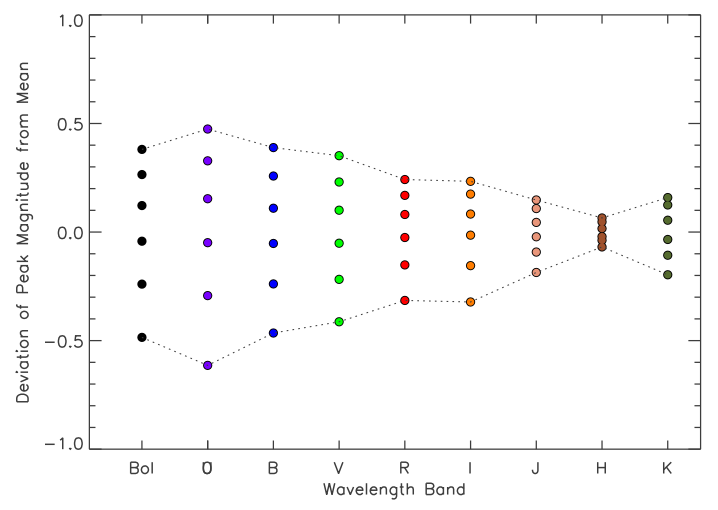

Figure 6: Dispersion in peak magnitude (measured at the first lightcurve maximum) as a function of wavelength band for SN Ia models with ${ }^{56} \mathrm{Ni}$ masses between 0.4 and $0.9 M_{\odot}($ Kasen 2006 [49]).

a function of wavelength band for SN Ia models with ${ }^{56} \mathrm{Ni}$ masses between 0.4 and $0.9 M_{\odot}$ [49].

It is important to obtain high quality spectra of SNe Ia (including NIR spectra, see [50]), since the spectra of SNe Ia have been shown to provide calibration relations that decrease the scatter of SNe Ia in the Hubble diagram, and make SNe Ia better distance indicators. The correlation between SN Ia spectroscopic features and luminosity has been found in the observational data (see, e.g., [51]). More recently, Bailey et al. (2009) [52] used the Nearby Supernova Factory spectrophotomery of $58 \mathrm{SNe}$ Ia to perform an unbiased search for flux ratios that correlate with SN Ia luminosity. They found that the $642 / 443 \mathrm{~nm}$ flux ratio is most strongly correlated with SN Ia absolute magnitudes. The correlation of SN Ia spectroscopic features and luminosity can be understood through comparing theoretical modeling with observational data. Hachinger et al. (2008) [53] found that the strength of the Si II $\lambda 5972$ line may be a very promising spectroscopic luminosity indicator for SNe Ia, with the correlation between Si II $\lambda 5972$ strength and luminosity resulting from the effect of ionization balance. 

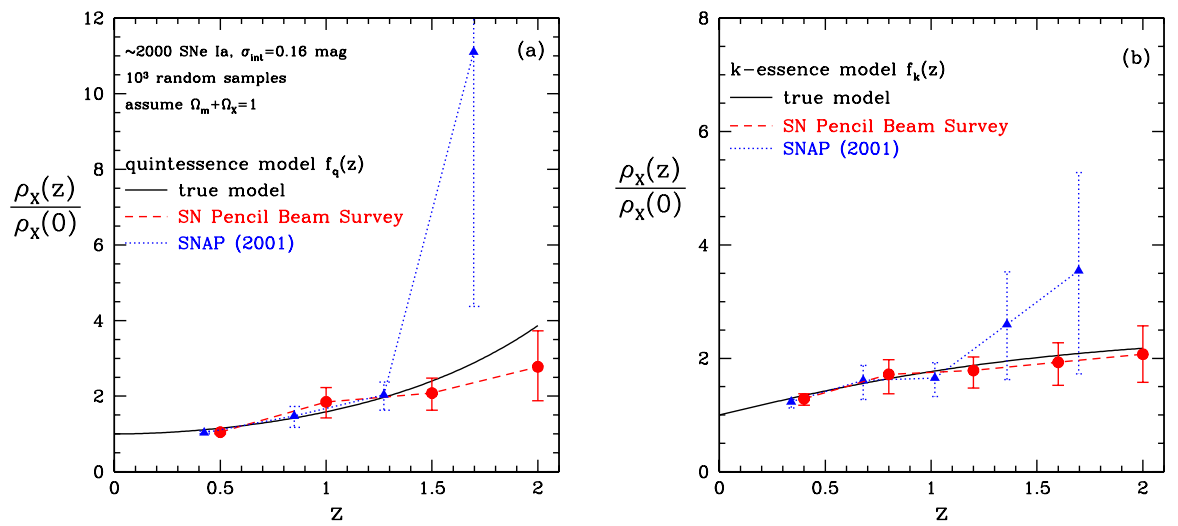

Figure 7: The comparison of an ultra deep supernova survey [55] with a much shallower survey in the reconstruction of the dark energy density $\rho_{X}(z)$ as a free function of cosmic time [54].

The key to the efficient use of SNe Ia for probing dark energy is to obtain the largest possible unbiased sample of SNe Ia at the greatest distances from the observer [54]. This is achieved by an ultra deep survey of the same areas in the sky every few days over at least one year [55]. Given the same observational resources, an ultra deep supernova survey is superior to a much shallower survey. A sufficiently deep supernova survey is required to reconstruct the dark energy density $\rho_{X}(z)$ as a free function of cosmic time (i.e., to measure $H(z)$ precisely, see Fig.7) [54].

\section{Galaxy clustering as dark energy probe}

Baryon acoustic oscillations (BAO) as a standard ruler. At the last scattering of CMB photons, the acoustic oscillations in the photon-baryon fluid became frozen, and imprinted their signatures on both the CMB (the acoustic peaks in the CMB angular power spectrum) and the matter distribution (the baryon acoustic oscillations in the galaxy power spectrum). Because baryons comprise only a small fraction of matter, and the matter power spectrum has evolved significantly since last scattering of photons, BAO are much smaller in amplitude than the CMB acoustic peaks, and are washed out on small scales. BAO in the observed galaxy power spectrum have the characteristic scale determined by the comoving sound horizon at the drag epoch (which occurred shortly after recombination), which is precisely measured by the CMB anisotropy data (see, e.g., [8]). Comparing the observed BAO scales with the expected values gives $H(z)$ in the radial direction, and $D_{A}(z)=r(z) /(1+z)$ (the angular diameter distance) in the transverse direction [56, 57]. Fig.8 shows the first detection of the BAO peak from a sample of the SDSS LRGs [58].

$\mathrm{BAO}$ represents only a fraction of the cosmological information contained in galaxy clustering data. A flux-limited galaxy redshift survey can allow us to measure the cosmic expansion history $H(z)$ through BAO only or the shape of the galaxy power spectrum $P(k)$ (which includes BAO as features) measured from the galaxy distribution, and the growth history of cosmic large scale structure $f_{g}(z)$ through independent measurements of redshift-space distortions and the bias factor between the distribution of galaxies and that of matter [19]. Assuming linear bias, the combination $f_{g}(z) \sigma_{8 m}(z)$ can be measured directly and used to test gravity [59]. 


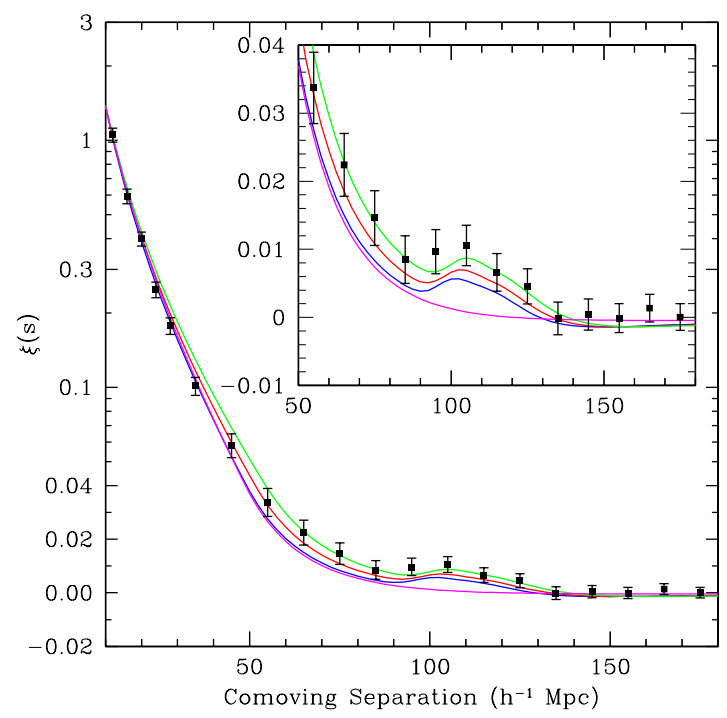

Figure 8: The spherically-averaged galaxy correlation function measured from the SDSS data, clearly showing a peak corresponding to the BAO scale at $\sim 100 h^{-1} \mathrm{Mpc}[58]$.

Current GC Measurements. The first simultaneous measurements of $H(z)$ and $D_{A}(z)$ from galaxy clustering data was made very recently by Chuang \& Wang (2012) [11], based on a Markov Chain Monte Carlo (MCMC) analysis of the two-dimensional two-point correlation function (2D 2PCF) they measured from the flux-limited sample of LRGs from the SDSS Data Release 7 [11]. Fig.9 shows the 2D 2PCF measured from the SDSS LRGs and a single LasDamas SDSS LRG mock catalog for comparison. The similarity between the data and the mock in the range of scales used (indicated by the shaded disk) is apparent. Due to the current limitations in the modeling of systematic effects, only the quasi-linear scale range of $s=40-120 h^{-1} \mathrm{Mpc}$ is used for a conservative estimate in this analysis. Chuang \& Wang (2012) [11] obtained

$$
H(z=0.35)=82.1_{-4.9}^{+4.8} \mathrm{~km} \mathrm{~s}^{-1} \mathrm{Mpc}^{-1}, \quad D_{A}(z=0.35)=1048_{-58}^{+60} \mathrm{Mpc}
$$

without assuming a dark energy model or a flat universe. Scaling $H(z)$ and $D_{A}(z)$ using $r_{s}\left(z_{d}\right)$ (the sound horizon at the drag epoch) in the MCMC analysis, they found that the derived measurements of

$$
H(0.35) r_{s}\left(z_{d}\right)=13020 \pm 530 \mathrm{~km} / \mathrm{s}, \quad r_{s}\left(z_{d}\right) / D_{A}(0.35)=0.1518 \pm 0.0062
$$

are nearly uncorrelated (with a normalized correlation coefficient of $r=-0.0584$ ), have tighter constraints and are more robust with respect to possible systematic effects. This is as expected, since $H(0.35) r_{s}\left(z_{d}\right)$ and $r_{s}\left(z_{d}\right) / D_{A}(0.35)$ correspond to the preferential redshift separation along the line of sight, and the preferential angular separation in the transverse direction respectively; these in turn arise from the BAO in the radial and transverse directions. The measurable preferential redshift and angular separations should be uncorrelated since they are independent degrees of freedom. The presence of the BAO (although only marginally visible in Fig.9) leads to tight and robust constraints on $H(0.35) r_{s}\left(z_{d}\right)$ and $r_{s}\left(z_{d}\right) / D_{A}(0.35)$. Since most of the constraining power in this analysis comes from fitting the overall shape of the galaxy correlation function on quasi-linear 

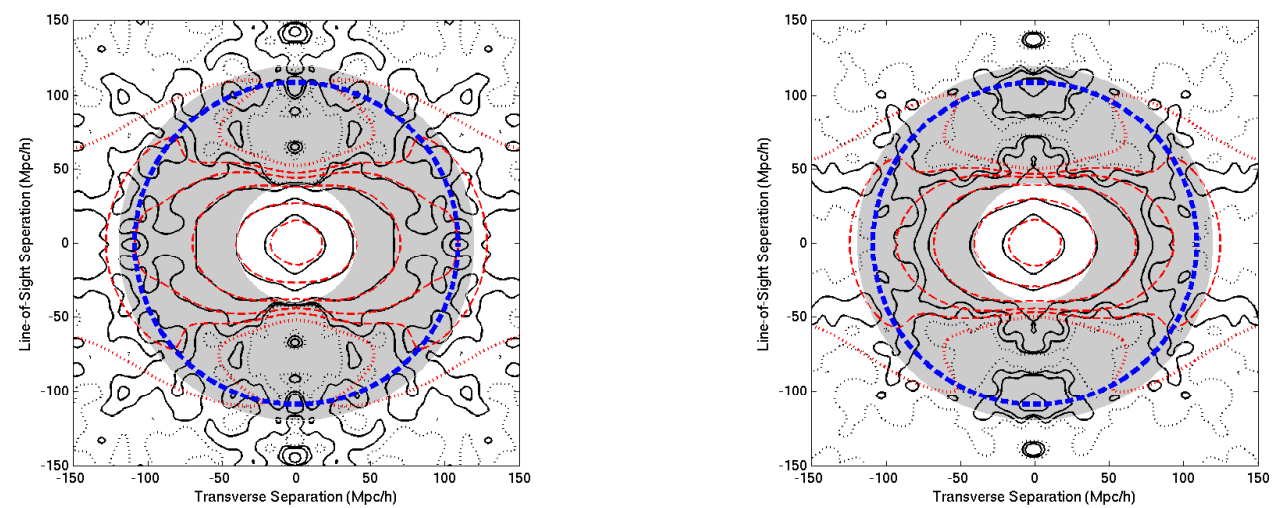

Figure 9: The two-dimensional two-point correlation function (2D 2PCF) measured from SDSS DR7 LRGs (left panel) and a LasDamas SDSS LRG mock catalog (right panel) in a redshift range $0.16<z<0.44$ (solid black contours), compared to a theoretical correlation function with parameters close to the best fit values in the likelihood analysis (dashed red contours). In both figures, the shaded disk indicates the scale range considered $\left(s=40-120 h^{-1} \mathrm{Mpc}\right)$ in this study. The thick dashed blue circle denotes the baryon acoustic oscillation scale. The observed 2D 2PCF has been smoothed by a Gaussian filter with rms variance of $2 h^{-1} \mathrm{Mpc}$ for illustration in these figures only; smoothing is not used in our likelihood analysis. The contour levels are $\xi=0.5,0.1,0.025,0.01,0.005,0$. The $\xi=0$ contours are denoted with dotted lines for clarity. [11]

scales, and not from fitting the BAO peaks, these measurements are galaxy clustering measurements (rather than BAO only measurements).

The constraints in Eq.(4.2) can be used to combine with CMB and any other cosmological data sets to constrain dark energy, as no priors were imposed that would affect the combined constraints $[11,60] . .^{5}$ These results have significant implications for future surveys in establishing the feasibility of measuring both $H(z)$ and $D_{A}(z)$ from galaxy clustering data.

Fig.10 shows the first results from the WiggleZ Dark Energy Survey obtained by Blake et al. (2011ab) [62, 63]. Note that Fig.10 (left panel) assumes fixed values of $\left(\Omega_{b} h^{2}, n_{S}, h, \sigma_{8}\right)$, and the background cosmological model is assumed to be known for Fig.10 (right panel). When dramatically larger data sets become available from the next generation galaxy redshift surveys, it will be possible to extract both distance and growth rate measurements simultaneously without imposing strong priors (see, e.g., [64]).

Possible systematic differences in different GC measurements. Ref.[7] showed that there may be systematic differences in different GC measurements. Fig.11 shows the 2D marginalized contours of $\left(w, \Omega_{m}, \Omega_{k}\right)$ for different GC measurements combined with CMB, $H_{0}$, and GRB data. The first row of Fig.11 compares the $H(z=0.35) r_{s}\left(z_{d}\right)$ and $r_{s}\left(z_{d}\right) / D_{A}(z=0.35)$ measurements by Chuang \& Wang (2012) [11] with their $d_{0.35}=r_{s}\left(z_{d}\right) / D_{V}(z=0.35)$ measurement (both from SDSS DR7 LRGs), as well as the $d_{0.2}$ and $d_{0.35}$ measurements by Percival et al. (2010) [65] from SDSS DR7 LRG and main galaxy samples and 2dFGRS, and the $d_{0.6}$ measurement by Blake et al. (2011) from the WiggleZ survey [68] combined with the $d_{0.106}$ measurement by Beutler et al. (2011) from 6dF GRS [69].

For the Chuang \& Wang (2012) [11] GC measurements (CW2 and CW1), the constraints on

\footnotetext{
${ }^{5}$ For the most recent results on the measurement of $H(z)$ and $D_{A}(z)$ from GC data, see [61].
} 

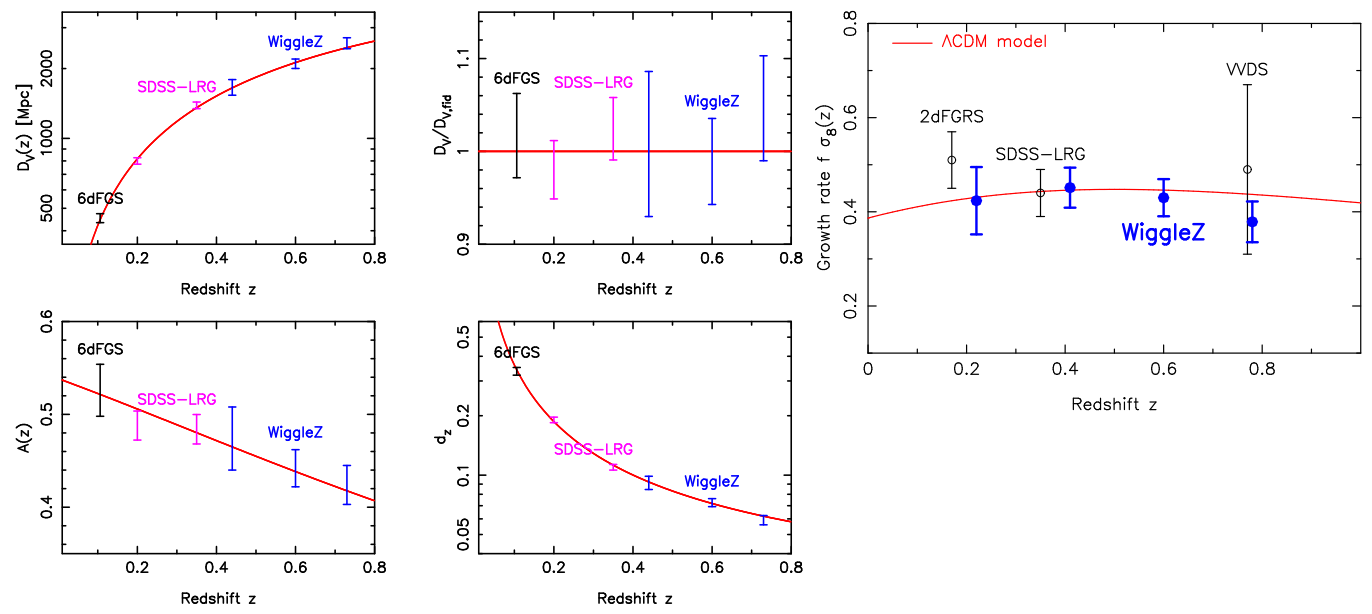

Figure 10: Left panel: Measurements of the baryon acoustic peak at redshifts $z=0.44,0.6$ and 0.73 in the galaxy correlation function of the final data set of the WiggleZ Dark Energy Survey. [62] Right panel: Measurements of $f_{g}(z) \sigma_{8 m}(z)$ from the WiggleZ Dark Energy Survey data. [63]
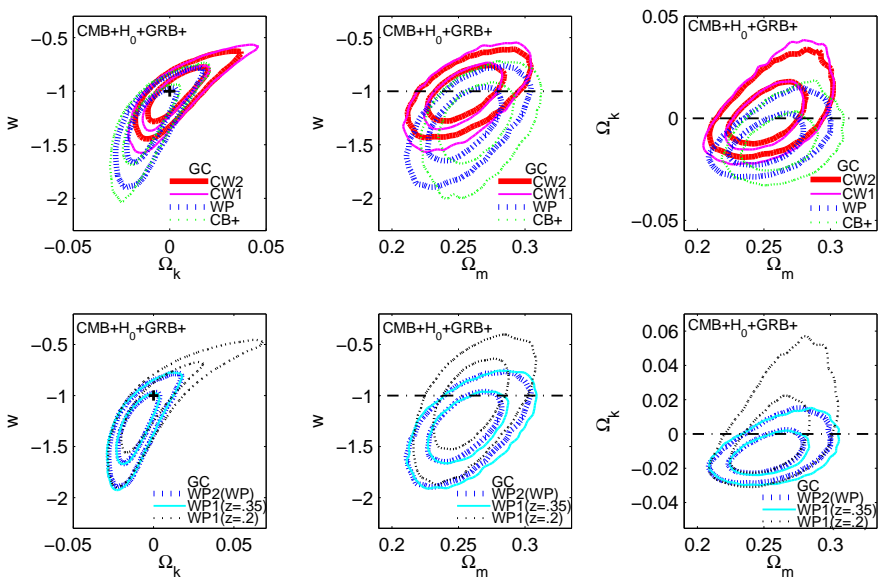

Figure 11: The 2D marginalized contours of $\left(w, \Omega_{m}, \Omega_{k}\right)$ for different galaxy clustering measurements combined with $\mathrm{CMB}, H_{0}$, and GRB data. The contours are at $68 \%$ and $95 \%$ confidence levels.

$w$ are tightened significantly by going from spherically-averaged data (CW1), i.e., $d_{0.35}$, to $2 \mathrm{D}$ data (CW2), i.e., $H(z=0.35) r_{s}\left(z_{d}\right)$ and $r_{s}\left(z_{d}\right) / D_{A}(z=0.35)$, as indicated by comparing the thin solid contours (CW1) to thick solid contours (CW2) in the first row of Fig.11. This is as expected, as more information from GC is included in CW2 compared to CW1. Both the Percival et al. (2010) GC measurements (WP) and the combined WiggleZ survey and 6dF GRS measurements (CB+) favor $w<-1$ (similar results were found by [67] using GC measurements from [65]), while the Chuang \& Wang (2012) [11] GC measurements favor $w=-1$.

The second row in Fig.11 compares the $d_{0.2}$ and $d_{0.35}$ measurements by Percival et al. (2010) [65] (WP2), with their measurements of $d_{0.2}$ and $d_{0.35}$ separately. Clearly, most of the constraining power on $w$ comes from $d_{0.35}$. While the $d_{0.2}$ measurement favors $w=-1$, the $d_{0.35}$ measurement favors $w<-1$. The measurements of $d_{0.35}$ by Chuang \& Wang (2012) [11] and Percival et al. (2010) [65] are similar in precision, but differ systematically: $d_{0.35}^{C W} \equiv r_{s}\left(z_{d}\right) / D_{V}(z=0.35)=$ $0.1161 \pm 0.0034$, while $d_{0.35}^{W P} \equiv r_{s}\left(z_{d}\right) / D_{V}(z=0.35)=0.1097 \pm 0.0036$. The lower measured value 

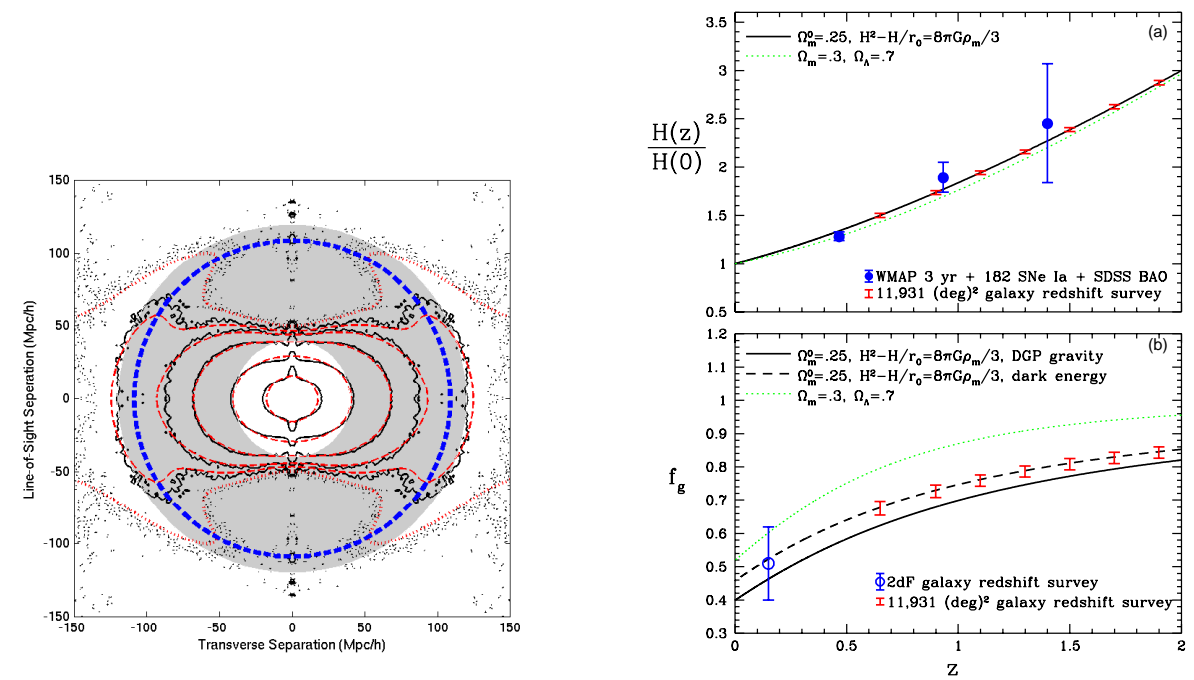

Figure 12: Left panel: The average two-dimensional two-point correlation function (2D 2PCF) measured from 160 LasDamas SDSS LRGfull mock catalogs (solid black contours), compared to a theoretical model with the input parameters of the LasDamas simulations (dashed red contours). The line types and contour levels are the same as in Fig.9 [11]. Right panel: Current and expected future measurements of the cosmic expansion history $H(z)=H_{0} E(z)$ and the growth rate of cosmic large scale structure $f_{g}(z)$ ). The future data correspond to a magnitude-limited NIR galaxy redshift survey covering $>10,000$ square degrees and $0.5<z<2$. [19]

of $d_{0.35}^{W P}$ implies a smaller $H(z=0.35)$, which in turn implies a more negative $w$. When combined with CMB, $H_{0}$, and GRB data, $d_{0.35}^{C W}$ favors $w=-1$, while $d_{0.35}^{W P}$ favors $w<-1$. Note that these two measurements used different methods to analyze GC data: Chuang \& Wang (2012) used the galaxy correlation function, while Percival et al. (2010) used galaxy power spectrum. It is not surprising that they lead to different distance measurements from GC. However, Montesano, Sanchez, \& Phleps (2012) [66] obtained results consistent with $w=-1$ using the galaxy power spectrum of the SDSS DR7 LRGs.

Future Prospects. The data contours in Fig.12 (left panel) gives a sense of the exquisite precision the galaxy 2D 2PCF can be measured when significantly larger data sets become available; it shows the averaged 2D 2PCF measured from 160 LasDamas mock catalogs compared with a theoretical model [11]. The contour levels are apparent in the measured 2D 2PCF even though no smoothing is used (in contrast to the noisy current data, see Fig.9); this is due to the reduction of shot noise achieved by averaging over 160 mock catalogs. Clearly, the 2D theoretical model used by [11] provides a reasonable fit to data on intermediate (and quasi-linear) scales.

A flux-limited galaxy redshift survey can allow us to measure both $H(z)$ and $f_{g}(z)[18,19]$. The measurement of $f_{g}(z)$ can be obtained through independent measurements of redshift-space distortion parameter $\beta=f_{g}(z) / b[70]$ and the bias parameter $b(z)$ (which describes how light traces mass) [18]. The parameter $\beta$ can be measured directly from galaxy redshift survey data by studying the observed redshift-space correlation function $[71,72]$. We can assume that the galaxy density perturbation $\delta_{g}$ is related to the matter density perturbation $\delta(\mathbf{x})$ as follows [73]: $\delta_{g}=b \delta(\mathbf{x})+$ $b_{2} \delta^{2}(\mathbf{x}) / 2$. The galaxy bispectrum is $\left\langle\boldsymbol{\delta}_{g \mathbf{k}_{1}} \delta_{g \mathbf{k}_{2}} \delta_{g \mathbf{k}_{1}}\right\rangle=(2 \pi)^{3}\left\{P_{g}\left(\mathbf{k}_{1}\right) P_{g}\left(\mathbf{k}_{2}\right)\left[J\left(\mathbf{k}_{1}, \mathbf{k}_{2}\right) / b+b_{2} / b^{2}\right]+c y c.\right\} \delta^{D}\left(\mathbf{k}_{1}+\right.$ 
$\left.\mathbf{k}_{2}+\mathbf{k}_{3}\right)$, where $J$ is a function that depends on the shape of the triangle formed by $\left(\mathbf{k}_{1}, \mathbf{k}_{2}, \mathbf{k}_{3}\right)$ in $\mathbf{k}$ space, but only depends very weakly on cosmology [74]. Ref.[74] developed the method for measuring $b(z)$ from the galaxy bispectrum, which was applied by [75] to the $2 \mathrm{dF}$ data. Independent measurements of $\beta(z)$ and $b(z)$ are very limited at present $[71,75]$; this will change dramatically in the near future.

The right panel of Fig.12 shows how well a flux-limited NIR galaxy redshift survey covering $>10,000$ square degrees and $0.5<z<2$ can constrain $H(z)$ and $f_{g}(z)$, compared with current data [19]. The bottom half of the right panel in Fig.12 shows the $f_{g}(z)$ for a modified gravity model (the DGP gravity model) with $\Omega_{m}^{0}=0.25$ (solid line), as well as a dark energy model that gives the same $H(z)$ for the same $\Omega_{m}^{0}$ (dashed line). The cosmological constant model from the top half of the right panel in Fig. 12 is also shown (dotted line). Clearly, current data can not differentiate between dark energy and modified gravity. A very wide and deep galaxy redshift survey provides measurement of $f_{g}(z)$ accurate to a few percent; this will allow an unambiguous distinction between dark energy models and modified gravity models that give identical $H(z)$ (see the solid and dashed lines in the bottom half of the right panel of Fig.12).

The systematic effects of BAO as a standard ruler are: bias between luminous matter and matter distributions, nonlinear effects, and redshift distortions [56]. Cosmological N-body simulations are required to quantify these effects [76]. Ref.[77] shows that nonlinear effects can be accurately taken into account. Ref.[78] shows that the BAO signal is boosted when bias, nonlinear effects, and redshift distortions are properly included in the Hubble Volume simulation. For a detailed discussion, see [6].

\section{Summary: Current Status and Future Prospects}

There are a large number of dark energy surveys that are ongoing, planned, or proposed. Ongoing projects include Carnegie Supernova Project (CSP), ESO Visible and Infrared Survey Telescope for Astronomy (VISTA) Surveys, Panoramic Survey Telescope \& Rapid Response System (PanSTARRS), Hobby-Eberly Telescope Dark Energy Experiment (HETDEX), and Sloan Digital Sky Survey (SDSS) III, and the Dark Energy Survey (DES) [79]. Selected future projects include Large Synoptic Survey Telescope (LSST) [80], and Euclid [64, 81]. Proposed future projects include the Wide-Field Infrared Survey Telescope (WFIRST), BigBOSS, and Square Kilometre Array (SKA) [82]. It is critical to remember that the challenge to solving the dark energy mystery will not be the statistics of the data obtained, but the tight control of systematic effects inherent in the data. A combination of all three most promising methods (SNe Ia, GC, and WL) should be used, each optimized by having its systematics minimized by design [83]. It is an exciting time in cosmology. We can expect to make ground-breaking discoveries about the nature of dark energy within the next decade or two.

I am grateful to Chris Blake, Daniel Eisenstein, Mario Hamuy, Dan Kasen, and Kevin Krisciunas for permission to use their figures in this proceeding paper. This work was supported in part by DOE grant DE-FG02-04ER41305.

\section{References}

[1] Riess A G et al., 1998, AJ, 116, 1009; Perlmutter S et al., 1999, ApJ, 517, 565 
[2] K. Freese et al., Nucl.Phys. B287, 797 (1987); A. Linde, in Three hundred years of gravitation, (Eds.: Hawking, S.W. and Israel, W., Cambridge Univ. Press, 1987), 604; P. J. E Peebles and B. Ratra, ApJ 325, L17 (1988); C. Wetterich, Nucl.Phys. B302, 668 (1988); J. A. Frieman, C. T. Hill, A. Stebbin, and I. Waga, PRL 75, 2077 (1995); R. Caldwell, R. Dave, and P. J. Steinhardt, PRL 80, 1582 (1998); L. Wang and P. J. Steinhardt, ApJ 508, 483 (1998).

[3] V. Sahni and S. Habib, PRL 81, 1766 (1998); L. Parker and A. Raval, PRD 60, 063512 (1999); C. Deffayet, Phys.Lett.B 502, 199 (2001); K. Freese and M. Lewis, Phys.Lett.B 540, 1 (2002); V. K. Onemli and R. P. Woodard, PRD 70, 107301 (2004).

[4] G. Dvali, G. Gabadadze, M. Porrati, PLB, 485, 208 (2000)

[5] Copeland, E. J., Sami, M., Tsujikawa, S., IJMPD, 15 (2006), 1753; Ruiz-Lapuente, P., Class. Quantum. Grav., 24 (2007), 91; Ratra, B., Vogeley, M. S., arXiv:0706.1565 (2007); Frieman, J., Turner, M., Huterer, D., ARAA, 46, 385 (2008); Caldwell, R. R., \& Kamionkowski, M., arXiv:0903.0866; Uzan, J.-P., arXiv:0908.2243; Y.-F. Cai, et al., Phys. Rep. 493, 1 (2010); Li, M., et al., 2011, arXiv1103.5870; D.H. Weinberg, et al. (2012), arXiv:1201.2434

[6] Wang, Y., Dark Energy, Wiley-VCH (2010)

[7] Wang, Y.; Chuang, C.-H.; Mukherjee, P., Phys. Rev. D 85, 023517 (2012); arXiv1109.3172

[8] Komatsu, E., et al. 2011, Astrophys.J.Suppl., 192, 18

[9] Conley, A., et al., 2011, Astrophys.J.Suppl., 192, 1

[10] A. G. Riess et al., ApJ 659, 98 (2007).

[11] Chuang, C.-H.; and Wang, Y., MNRAS, 426, 226 (2012); arXiv:1102.2251

[12] For recent reviews, see H. Hoekstra, \& B.Jain, Ann.Rev.Nucl.Part.Sci., 58, 99 (2008); D. Munshi, et al., Physics Reports, 462, 67 (2008); A. Heavens, arXiv:1109.1121

[13] For a recent review, see S. W. Allen, A. E. Evrard, A. B. Mantz, arXiv:1103.4829

[14] Wang, Y., 2008, PRD, 78, 123532

[15] See, e.g., Schaefer, B. E., 2007, ApJ, 660, 16; Simon, J.; Verde, L.; \& Jimenez, R. 2005, PRD, 71, 123001; Daly, R. A., et al., 2009, ApJ, 691, 1058

[16] Riess, A. G, et al., 2011, ApJ, 730, 119

[17] Wang, Y.; and Mukherjee, P., PRD, 76, 103533 (2007)

[18] Guzzo L et al., Nature, in press (2008)

[19] Wang, Y., Journal of Cosmology and Astroparticle Physics, 05, 021 (2008).

[20] Chevallier, M., \& Polarski, D. 2001, Int. J. Mod. Phys. D10, 213

[21] Wang, Y.; and Garnavich, P., ApJ, 552, 445 (2001)

[22] M. Tegmark, Phys. Rev. D66, 103507 (2002); Y. Wang, \& K. Freese, Phys.Lett. B632, 449 (2006)

[23] Y. Wang; J.M. Kratochvil; A. Linde; M. Shmakova, JCAP, 12, 006 (2004)

[24] Y. Wang, \& M. Tegmark, Phys. Rev. Lett., 92, 241302 (2004)

[25] H.B. Sandvik; M. Tegmark; M. Zaldarriaga; I. Waga, Phys. Rev. D 69, 123524 (2004) 
[26] J-P. Uzan, and F. Bernardeau, Phys. Rev. D64, 083004 (2001);

H. F. Stabenau and B. Jain, PRD, 74, 084007; ( 2006)

A. F. Heavens, T.D. Kitching, L. Verde, astro-ph/0703191; J.-P. Uzan, Gen. Relat. Grav. 39, 307

(2007) P. Zhang; M. Liguori;R. Bean; S. Dodelson, Phys.Rev.Lett. 99, 141302 (2007)

[27] A. Lue, R. Scoccimarro, and G. D. Starkman, PRD 69, 124015 (2004); A. Lue, Physics Report 423, 1 (2006).

[28] Y.-S. Song, I. Sawicki, and W. Hu, PRD, 75, 064003 (2007); K. Koyama, arXiv:0709.2399

[29] Bhattacharya, S.; Kosowsky, A.; Newman, J. A.; Zentner, A. R. PRD, 83, 043004 (2011)

[30] Y. Wang, \& M. Tegmark, Phys. Rev. D 71, 103513 (2005)

[31] M. M. Phillips, ApJ 413, L105 ( 1993);

[32] A.G. Riess, W.H. Press, \& R.P. Kirshner, ApJ, 438, L17 (1995)

[33] G. Goldhaber, et al, ApJ, 558, 359 (2001)

[34] Wheeler, J. C., AAPT/AJP Resource Letter, Am. J. Phys., 71 (2003), astro-ph/0209514

[35] See, e.g., S. Bongard, et al., ApJ, 647, 513 (2006); D. Branch, et al., PASP, 118, 560 (2006); Y. Wang, and N. Hall, MNRAS, 389, 489 (2008).

[36] M. Hamuy, et al., AJ, 112, 2398 (1996)

[37] J.A. Cardelli; G.C. Clayton; \& J.S. Mathis, ApJ, 345, 245 (1989)

[38] A.N. Aguirre, ApJ, 525, 583 (1999)

[39] R. Kantowski, T. Vaughan, \& D. Branch, ApJ, 447, 35 (1995); J. A. Frieman, Comments Astrophys., 18, 323 (1997); J. Wambsganss, R. Cen, G. Xu, \& J.P. Ostriker, ApJ, 475, L81 (1997); D.E. Holz, ApJ, 506, L1 (1998); Y. Wang, ApJ, 525, 651 (1999)

[40] A.N. Aguirre, and Z. Haiman, ApJ, 532, 28 (2000)

[41] P. Zhang, and P.S. Corasaniti, ApJ, 657, 71 (2007)

[42] Conley, A., et al. 2007, ApJ, 664, L13

[43] Y. Wang; D.E. Holz; \& D. Munshi, ApJ, 572, L15 (2002)

[44] Y. Wang, ApJ, 536, 531 (2000); Y. Wang; \& P. Mukherjee, ApJ, 606, 654 (2004).

[45] D. Branch; S. Perlmutter; E. Baron; \& P. Nugent, astro-ph/0109070

[46] K. Krisciunas; M.M. Phillips; N.B. Suntzeff, ApJ, 602, L81 (2004)

[47] M. M. Phillips; P. Garnavich; Y. Wang; et al., SPIE proceedings (2006), astro-ph/0606691

[48] W. Michael Wood-Vasey, et al., arXiv:0711.2068, ApJ submitted (2007)

[49] Kasen, D., 2006, ApJ, 649, 939

[50] Marion, G. H., et al. 2006, ApJ, 645, 1392; 2009, AJ, 138, 727

[51] Garnavich, P. M., et al. 2004, ApJ, 613, 1120

[52] Bailey, S. et al. 2009, arXiv:0905.0340

[53] Hachinger, S., et al. 2008, MNRAS, 389, 1087

[54] Y. Wang, \& G. Lovelace, ApJ Letter, 562, L115 (2001) 
[55] Y. Wang, ApJ, 531, 676 (2000), astro-ph/9806185

[56] Blake, C., \& Glazebrook, K, 2003, ApJ, 594, 665; Seo, H., \& Eisenstein, D. J., 2003, ApJ, 598, 720

[57] See, e.g., M. White, Astropart. Phys. 24, 334 (2005); G. Huetsi, A\&A 449, 891 (2006); Y. Wang, ApJ 647, 1 (2006); R. Angulo et al., astro-ph/0702543. (2007).

[58] D. Eisenstein et al., ApJ 633, 560 (2005);

[59] Song, Y.-S.; Percival, W. J., 2009, JCAP, 10, 004

[60] Chuang, Chia-Hsun; Wang, Yun; Hemantha, Maddumage Don P., MNRAS, 423, 1474 (2012); arXiv1008.4822

[61] Reid, B.A., et al. (2012), arXiv:1203.6641; Chuang, C.H., \& Wang, Y., arXiv:1205.5573; Xu, X., et al. (2012), arXiv:1206.6732; Chuang, C.H., \& Wang, Y., arXiv:1209.0210

[62] Blake, C., et al., 2011, MNRAS, 418, 1707

[63] Blake, C., et al., 2011, MNRAS, 415, 2876

[64] Laureijs, R., et al., arXiv1110.3193, Euclid Definition Study Report

[65] Percival, W.J., et al. 2010, MNRAS, 401, 2148

[66] Montesano, F.; Sanchez, A. G.; Phleps, S. 2012, MNRAS, 421, 2656

[67] Escamilla-Rivera, C.; et al., 2011, JCAP, 09, 003; Li, X.-D., et al., JCAP 07 (2011) 011

[68] Blake, C., et al. 2011, MNRAS, 415, 2892

[69] Beutler, F., et al., 2011, arXiv:1106.3366, MNRAS, in press

[70] N. Kaiser, MNRAS, 227, 1 (1987)

[71] E. Hawkins et al., MNRAS, 346, 78 (2003)

[72] M. Tegmark et al., PRD 74, 123507 (2006); N. P. Ross et al., MNRAS 381, 573 (2007); J. da Angela et al., astro-ph/0612401.

[73] J. N. Fry and E. Gaztanaga, ApJ, 413, 447 ( 1993)

[74] S. Matarrese, L. Verde, and A. F. Heavens, MNRAS, 290, 651 ( 1997)

[75] L. Verde et al., MNRAS, 335, 432 (2002)

[76] Angulo, R., et al. 2005, Mon.Not.Roy.Astron.Soc.Lett., 362, L25-L29; Seo, H., \& Eisenstein, D. J., 2005, ApJ, 633, 575; V. Springel, et al., Nature, 435, 629 (2005); White, M. 2005, Astropart.Phys. 24, 334

[77] Jeong, D., \& Komatsu, E., 2006, ApJ, 651, 619; M. Crocce, and R. Scoccimarro, arXiv:0704.2783; R. E. Smith, R. Scoccimarro, and R. K. Sheth, astro-ph/0703620; K. Heitmann, et al., ApJ, 705, 156 (2009); ApJ, 715, 104 (2010)

[78] R. S. Koehler, P. Schuecker, and K. Gebhardt, A\&A, 462, 7 (2007)

[79] See http://www.ociw.edu/csp/, http://www.vista.ac.uk/index.html, http://pan-starrs.ifa.hawaii.edu/public/, http://www.as.utexas.edu/hetdex/, http://www.sdss.org/, http://www.darkenergysurvey.org/.

[80] See LSST Science Collaborations, 2009, arXiv0912.0201; http://www.1sst.org/

[81] See http://www.euclid-emc.org/

[82] See http://wfirst.gsfc.nasa.gov/, http://bigboss.1bl.gov/, and http://www. skatelescope.org/

[83] Y. Wang et al., BAAS v36, n5;1560 (2004); A. Crotts et al., astro-ph/0507043 (2005); E. Cheng and Y. Wang et al., Proc. of SPIE Vol. 6265, 626529 (2006). 